\title{
Hyaluronan suppresses lidocaine-induced apoptosis of human chondrocytes in vitro by inhibiting the p53- dependent mitochondrial apoptotic pathway
}

\author{
Yoon-Jin LEE ${ }^{1}$, Soo A KIM ${ }^{2, *}$, Sang-Han LEE ${ }^{1}$ \\ ${ }^{1}$ Department of Biochemistry, ${ }^{2}$ Department of Physical Medicine and Rehabilitation, Soonchunhyang University College of Medicine, \\ Cheonan Hospital, Cheonan 330-721, Korea
}

\begin{abstract}
Aim: Intra-articular injection of local anesthetics (LAs) is a common procedure for therapeutic purposes. However, LAs have been found toxic to articular cartilage, and hyaluronan may attenuate this toxicity. In this study we investigated whether hyaluronan attenuated lidocaine-induced chondrotoxicity, and if so, to elucidate the underlying mechanisms.

Methods: Human chondrocyte cell line SW1353 and newly isolated murine chondrocytes were incubated in culture medium containing hyaluronan and/or lidocaine for $72 \mathrm{~h}$. Cell viability was evaluated using MTT assay. Cell apoptosis was detected with DAPI staining, caspase 3/7 activity assay and flow cytometry. Cell cycle distributions, ROS levels and mitochondrial membrane potential $(\Delta \Psi \mathrm{m})$ were determined using flow cytometry. The expression of p53 and p53-regulated gene products was measured with Western blotting. Results: Lidocaine $(0.005 \%-0.03 \%)$ dose-dependently decreased the viability of SW1353 cells. This local anesthetic $(0.015 \%$, $0.025 \%$ ) induced apoptosis, $\mathrm{G}_{2} / \mathrm{M}$ phase arrest and loss of $\Delta \Psi \mathrm{m}$, and markedly increased ROS production in SW1353 cells. Hyaluronan $(50-800 \mu \mathrm{g} / \mathrm{mL})$ alone did not affect the cell viability, but co-treatment with hyaluronan $(200 \mu \mathrm{g} / \mathrm{mL})$ significantly attenuated lidocaine-induced apoptosis and other abnormalities in SW1353 cells. Furthermore, co-treatment with lidocaine and hyaluronan significantly decreased the levels of p53 and its transcription targets Bax and p21 in SW1353 cells, although treatment with lidocaine alone did not significantly change these proteins. Similar results were obtained in ex vivo cultured murine chondrocytes. Conclusion: Hyaluronan suppresses lidocaine-induced apoptosis of human chondrocytes in vitro through inhibiting the p53-dependent mitochondrial apoptotic pathway.
\end{abstract}

Keywords: hyaluronan; lidocaine; intra-articular injection; osteoarthritis; chondrocytes; apoptosis; oxidative damage; mitochondria; p53

Acta Pharmacologica Sinica (2016) 37: 664-673; doi: 10.1038/aps.2015.151; published online 4 Apr 2016

\section{Introduction}

The intra-articular injection of local anesthetics (LAs), including lidocaine and bupivacaine, has commonly been used for the evaluation of atypical pain, as well as the temporary amelioration of pain in patients with osteoarthritis; it is generally accepted as safe. However, there are a number of reports concerning the toxic effects of LAs on cartilage and muscle ${ }^{[1,2]}$, in addition to the overt cartilage damage observed at the time of surgery. There is evidence supporting a chondrotoxic effect of LAs on articular chondrocytes from several recent studies that have demonstrated detrimental effects of LAs on chondrocyte viability and cartilage histology ${ }^{[3-5]}$. A small number of

\footnotetext{
${ }^{*}$ To whom correspondence should be addressed.

E-mail sooapmr@schmc.ac.kr

Received 2015-9-17 Accepted 2015-12-20
}

studies have attempted to establish the mechanisms by which LAs induce chondrotoxicity. It has been shown that LAs may cause mitochondrial dysfunction, inhibition of voltage-gated $\mathrm{Na}^{+}$and $\mathrm{Ca}^{2+}$ channels, or potassium channel blockade ${ }^{[6-8]}$. With respect to the genotoxic potential of LAs, reports in the literature remain both scarce and controversial. Nai et al have reported that no genotoxicity effects are observed after repetitive exposure to any LA in rats ${ }^{[9]}$. In contrast, it has also been reported that lidocaine may induce genotoxic effects, such as point mutations, and its metabolite, 2,6-dimethylaniline, may be carcinogenic in rats ${ }^{[10]}$. Recently, Grishko et al have found that the chondrotoxicity of LAs, especially those LAs associated with the induction of apoptosis, is related to mitochondrial dysfunction resulting from mitochondrial DNA damage $^{[11]}$.

Mitochondria are the main source of reactive oxygen spe- 
cies (ROS) and the major target of ROS-induced damage ${ }^{[12]}$. Increased levels of ROS may lead to DNA damage, after which the cell cycle checkpoints and cell death signals are activated, thus halting cell growth and eliminating multiplication of the genetically altered cells. Damaged cells either stop DNA replication at $G_{1}$ or $G_{2}$ phase to facilitate DNA repair before DNA synthesis or mitosis or induce apoptosis to eliminate the excessively damaged cells. The activation of the apoptotic pathway is a safeguard to remove irreparably damaged cells. The p53 protein plays a critical role in arresting the cycles of damaged cells at these checkpoints and inducing apoptosis by transmitting signals associated with antiproliferative cellular responses $^{[13]}$.

Hyaluronic acid, also called hyaluronan, is an anionic, nonsulfated glycosaminoglycan composed of 2000-25000 repeating disaccharides of $\mathrm{N}$-acetylglucosamine and glucuronic acid units. Hyaluronan is an essential component of the extracellular matrix and is involved in important physiological functions, such as cell-to-cell identification, cellular cohesion, growth regulation, and antioxidant capacity ${ }^{[14]}$. Its combination with LAs may also ameliorate apoptotic cell death and enhance cell survival in chondrocytes ${ }^{[15,16]}$. However, the precise mechanisms of hyaluronan actions, especially those associated with cytoprotection, have not been firmly established, despite its widespread use in outpatient clinics. Thus, a better understanding of chondrocyte responses to hyaluronan would be useful for unveiling the specific molecular pathways that may lead to chondroprotection. The goals of this study were to determine whether hyaluronan protects against chondrotoxicity after exposure to the LA lidocaine and, if so, to find the biomolecules responsible for the mechanism of enhanced survival in the human chondrocyte cell line SW1353 and in murine primary chondrocytes. In the present study, we found that sodium hyaluronate exerts a protective influence against lidocaine-induced apoptosis, which is related to the inhibition of mitochondrial oxidative damage and the resultant decrease in the p53 protein level.

\section{Materials and methods}

\section{Cell culture and treatment}

The following were obtained from Sigma-Aldrich Co (St Louis, MO, USA): 3-(4,5 dimethylthiazol-2-yl)-2,5-diphenyltetrazolium bromide (MTT), sodium dodecyl sulfate, rhodamine-123, propidium iodide (PI), 4'-6-diamidino-2-phenylindole (DAPI), and an antibody against $\beta$-actin. Antibodies against Bax, Bcl2 , caspase-3, and cleaved caspase-3 were obtained from Cell Signaling Technology (Beverly, MA, USA). Antibodies against p53 and p21, and an enhanced chemiluminescence (ECL) kit were purchased from Santa Cruz Biotechnology (Santa Cruz, CA, USA). Lidocaine $\mathrm{HCl}$ hydrate was obtained from Huons Co Ltd (Jecheon, Korea). BDDE-cross-linked hyaluronic acid was obtained from LG Life Sciences (Iksan, Korea). The human chondrocyte cell line SW1353 was obtained from the American Type Culture Collection (ATCC, Manassas, VA, USA). For murine chondrocyte culture, 3 litters (30 animals) of 6-day-old newborn mice were obtained from KOATECH
(Pyeongtaek, Korea). This study was approved by the animal institutional review board of Soonchunhyang University regarding the experiments involving rodents (Approval № SCH-15-0035). Primary mouse articular chondrocytes were isolated from the femoral condyles of newborn mice after collagenase $\mathrm{D}$ digestion $\left(3 \mathrm{mg} / \mathrm{mL}, 1 \mathrm{~h}\right.$ at $\left.37^{\circ} \mathrm{C}\right)$, as described previously ${ }^{[17]}$. They were used between passages 2 and 3 . The SW1353 cells and mouse primary articular chondrocytes were maintained in DMEM supplemented with $10 \%$ fetal bovine serum, $1 \mathrm{mmol} / \mathrm{L}$ glutamine, 100 units of penicillin/mL, and $100 \mu \mathrm{g}$ of streptomycin $/ \mathrm{mL}$. The cells were grown to $70 \%$ confluence in a monolayer culture in this medium for $24 \mathrm{~h}$ before treatment. They were then harvested with trypsin for flow cytometry, DNA fragmentation, and western blot analyses.

\section{MTT assay}

The extent of cytotoxicity was determined by MTT assays. Cells $\left(5 \times 10^{3}\right.$ cells/well) were seeded in 48 -well microtiter plates and then treated for 3 days, after which they were exposed to MTT $(0.1 \mathrm{mg} / \mathrm{mL})$ for an additional $4 \mathrm{~h}$. The absorbance of each cell was measured by a GloMax-Multi Microplate Multimode Reader (Promega, Madison, WI, USA).

\section{DAPI staining}

Nuclear condensation and fragmentation was observed by nucleic acid staining with DAPI. The SW1353 chondrocyte cells were treated with lidocaine and sodium hyaluronate, alone or in combination, harvested by trypsinization, and fixed in $100 \%$ methanol at room temperature for $20 \mathrm{~min}$. The cells were spread on slides, stained with DAPI solution $(2 \mu \mathrm{g} / \mathrm{mL})$, and analyzed under a FluoView confocal fluorescent microscope (FluoView FV10i; Olympus Corporation, Tokyo, Japan).

\section{Cell cycle analysis}

Trypsinized cells were pelleted by centrifugation and fixed in $70 \%$ ice-cold ethanol overnight at $-20^{\circ} \mathrm{C}$; they were then treated with DNase-free RNase A $(150 \mu \mathrm{g} / \mathrm{mL})$ and propidium iodide $(20 \mu \mathrm{g} / \mathrm{mL})$. DNA content was analyzed by flow cytometry using a MACSQuant Analyzer (Miltenyi Biotec, $\mathrm{GmbH}$, Bergisch Gladbach, Germany). The samples were analyzed with a flow cytometry machine to identify apoptotic, necrotic, and viable cells.

\section{Apoptosis assay}

The apoptotic cell distribution was determined using a Muse Annexin V and Dead Cell kit in accordance with the manufacturer's protocol (Merck Millipore, Darmstadt, Germany). In brief, cells were collected in culture medium, mixed with the Muse Annexin V and Dead Cell Reagent, and analyzed using a Muse Cell Analyzer (Merck Millipore).

\section{Caspase- $3 / 7$ activity}

The activation of caspase- $3 / 7$ was quantified with the ApoTox-Glo Triplex Assay kit in accordance with the manufacturer's protocol (Promega). Cells were seeded in 96-well microtiter plates and then treated with various concentrations of 
sodium hyaluronate and lidocaine. After $72 \mathrm{~h}$, the cells were incubated with substrate containing Caspase-Glo ${ }^{\circledR} 3 / 7$ assay buffer for $30 \mathrm{~min}$. Caspase-3/7 activities were calculated after the detection of luminescence by a GloMax-Multi Microplate Multimode Reader.

\section{Measurement of ROS levels}

Intracellular ROS levels were measured using DCF-DA. In short, cells were incubated with serum-free medium containing $10 \mu \mathrm{mol} / \mathrm{L}$ of DCF-DA for $30 \mathrm{~min}$ at $37^{\circ} \mathrm{C}$ in the dark. Next, the cells were washed twice with PBS, trypsinized, resuspended, and then immediately analyzed using a MACSQuant Analyzer (Miltenyi Biotec). A $530 \mathrm{~nm}$ band-pass filter was used to detect DCF-DA fluorescence, and each determination was based on the mean fluorescence intensity of 10000 cells.

\section{Mitochondrial membrane potential (MMP) disruption assay}

SW1353 cells were seeded in 6-well plates and incubated with different concentrations of sodium hyaluronate and lidocaine for $72 \mathrm{~h}$. Then, the cells were harvested and washed twice with PBS; the cells were subsequently stained with fresh medium containing rhodamine-123 (final concentration: $30 \mathrm{nmol} / \mathrm{L}$ ) at $37^{\circ} \mathrm{C}$ for $30 \mathrm{~min}$. The fluorescence intensity of the cells was analyzed by flow cytometry using a MACSQuant Analyzer (Miltenyi Biotec).

\section{Western blot analysis}

Western blot analyses were performed using cell lysate, as described previously ${ }^{[12]}$. Cell lysate containing $40 \mu \mathrm{g}$ of protein was separated on NuPAGE $4 \%-12 \%$ Bis-Tris polyacrylamide gels (Invitrogen, Carlsbad, CA, USA) and then electrophoretically transferred to Immuno-Blot PVDF membranes. The signals were visualized by means of an ECL detection kit using X-ray films. The blots were then stripped using a stripping buffer $(100 \mathrm{mmol} / \mathrm{L} \beta$-mercaptoethanol, $2 \%$ SDS, and $62.5 \mathrm{mmol} / \mathrm{L}$ Tris-HCl, $\mathrm{pH}$ 6.7) and re-probed with anti- $\beta$ actin as a loading control.

\section{siRNA-mediated gene silencing}

RNA interference of p53 was performed using a p53-targeting small interfering RNA (siRNA) duplex from Invitrogen (Oligo ID: HSS186390). In brief, cells were seeded in 6-well and 48-well plates and transfected at 50\% confluency with siRNA duplexes using Lipofectamine RNAiMAX (Invitrogen).

\section{Statistical analysis}

Statistical comparisons were performed using one-way analysis of variance (ANOVA) followed by Tukey's post hoc correction method for multiple comparisons, using SPSS version 17.0 (SPSS, Inc, Chicago, IL, USA). The data were expressed as the mean \pm SEM. Significant differences were identified by values of $P<0.05$.

\section{Results}

Sodium hyaluronate inhibits lidocaine-induced chondrotoxicity

To determine the effective doses for sodium hyaluronate treatment, SW1353 chondrocyte cells were treated with increasing concentrations of sodium hyaluronate and lidocaine, alone or in combination, for $72 \mathrm{~h}$. The cells were then subjected to an MTT assay. The exposure of cells to sodium hyaluronate at concentrations up to $800 \mu \mathrm{g} / \mathrm{mL}$ resulted in little effect on cell viability (Figure 1A), whereas lidocaine inhibited viability in a dose-dependent manner (Figure 1B). Under the combined treatment of the two compounds, lidocaine-induced cytotoxicity was clearly prevented by sodium hyaluronate. The protective effect of sodium hyaluronate against lidocaine toxicity was evident after $24 \mathrm{~h}$ of treatment (Figure 1C).

Sodium hyaluronate protects chondrocytes against lidocaineinduced apoptotic cell death

Subsequently, the morphological changes of the SW1353 cells in response to lidocaine and sodium hyaluronate, alone or in combination, were observed. At the end of the treatments, no characteristic features of the cells were observed (Figure 2A). During the 72 -h exposure to lidocaine $(0.015 \%$ and $0.025 \%)$, the cells reached approximately $70 \%$ and $60 \%$ confluency,
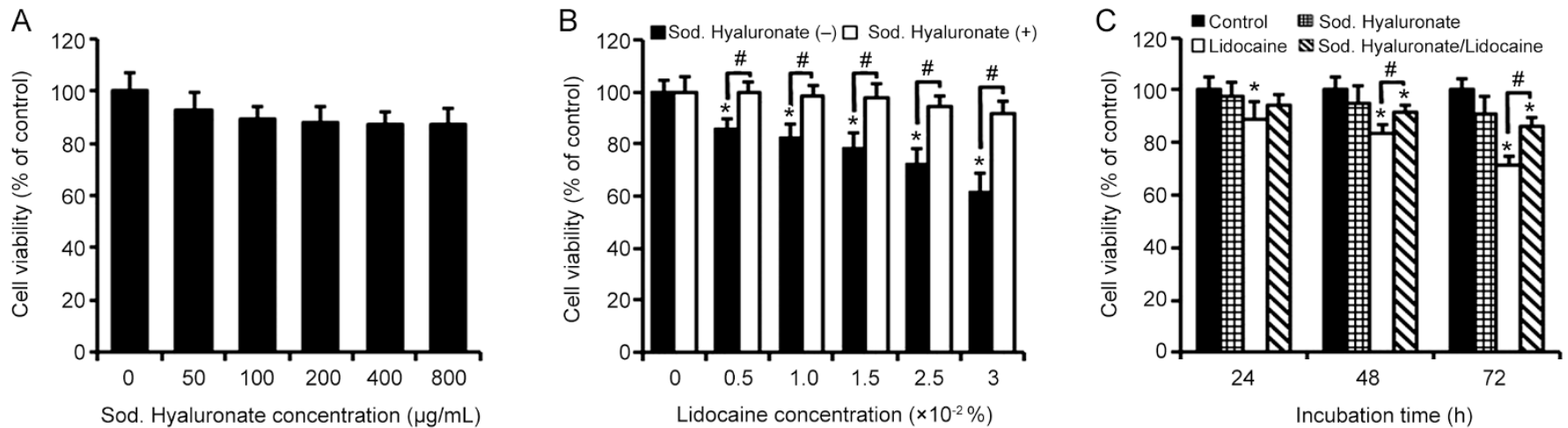

Figure 1. The effects of sodium hyaluronate and lidocaine, alone or in combination, on SW1353 cell proliferation. (A) Cells were incubated with the indicated concentrations of sodium hyaluronate for $72 \mathrm{~h}$. (B) Cells were incubated with sodium hyaluronate $(200 \mu \mathrm{g} / \mathrm{mL})$ and the indicated concentrations of lidocaine, alone or in combination, for $72 \mathrm{~h}$. (C) Cells were incubated with sodium hyaluronate $(200 \mu \mathrm{g} / \mathrm{mL})$ and lidocaine $(0.025 \%)$, alone or in combination, for the indicated times. Mean \pm SD. $n=3$. ${ }^{*} P<0.05$ vs the control. ${ }^{~} P<0.05$ between the two indicated groups. 
A
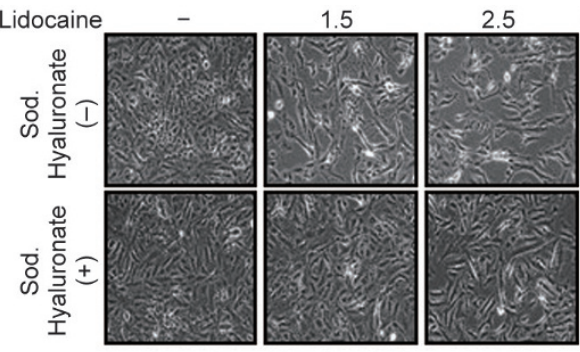

$\left(\times 10^{-2} \%\right)$

C

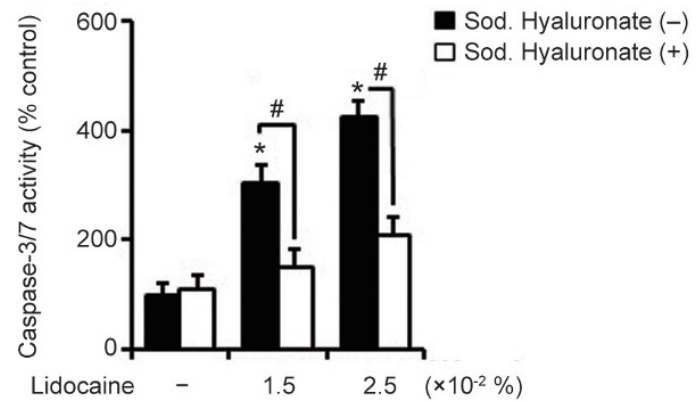

B

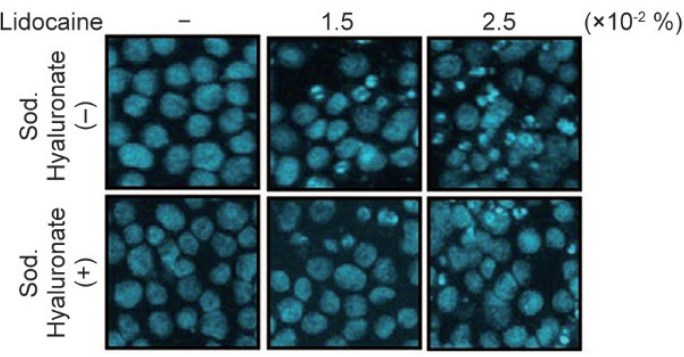

D

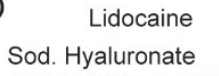



Figure 2. The effects of sodium hyaluronate and lidocaine, alone or in combination, on cell morphology, nuclear fragmentation, and caspase-3 activity in SW1353 cells. Cells were treated with sodium hyaluronate $(200 \mu \mathrm{g} / \mathrm{mL})$ and lidocaine $(0.015 \%$ and $0.025 \%)$, alone or in combination, for $72 \mathrm{~h}$. (A) Cell morphology was examined under phase-contrast microscopy. (B) Nuclear morphology was examined after staining cells with DAPI (2 $\mu \mathrm{g} / \mathrm{mL}$ ). (C) Caspase-3/7 activity was measured by using an ApoTox-Glo Triplex Assay. (D) Whole cell lysates were then analyzed by Western blotting with anticaspase-3 and anti-cleaved caspase-3. Mean \pm SD. $n=3$. " $P<0.05$ vs the control. ${ }^{*} P<0.05$ between the two indicated groups.

respectively. In contrast, the cells co-treated with lidocaine and sodium hyaluronate proliferated rapidly and reached approximately $85 \%-90 \%$ confluency. To determine whether lidocaine induced apoptosis in this cell line, the cells were stained with DAPI and visualized under a confocal microscope, which enabled the identification of several hallmarks of apoptosis. As shown in Figure 2B, the lidocaine treatment led to an increase in nuclear condensation and fragmentation compared with the controls, whereas the addition of sodium hyaluronate caused a statistically significant reduction in apoptosis compared with the lidocaine-treated group.

Caspase- 3 is the final enforcer of caspases in apoptosis, and its cleavage is a hallmark of the activation of apoptotic cell death. To examine the activation of caspases in apoptosis mediated by lidocaine in the SW1353 cells, caspase-3/7 activity was assessed in cell cytosolic extracts. The lidocaine treatment induced a concentration-dependent increase in caspase-3/7 activity (Figure 2C), as well as an enhanced cleavage of procaspase-3 (Figure 2D).

In the flow cytometry analysis, the occurrence of a sub- $\mathrm{G}_{0} /$ $\mathrm{G}_{1}$ peak, indicative of apoptosis, was detected in the cultures treated with final concentrations $0.015 \%$ and $0.025 \%$ of lidocaine (Figure 3A). Co-treatment with lidocaine and sodium hyaluronate significantly reduced the proportion of cells with hypodiploid amounts of DNA. With increasing lidocaine concentration, the percentage of cells in the $G_{1}$ and $S$ phases decreased, and those in the $G_{2} / M$ phase increased; these results indicated arrest of the cell cycle. The occurrence of the $\mathrm{G}_{2} / \mathrm{M}$ arrest was accompanied by increased annexin V-PE binding to the outer leaflet of the plasma membrane (Figure 3B). The proportion of cells undergoing apoptosis at early and late phases, increased with rising concentrations of lidocaine. Thus, following lidocaine treatment, the concurrence of the $\mathrm{G}_{2} / \mathrm{M}$ arrest and apoptosis were significantly attenuated by co-treatment with sodium hyaluronate.

Sodium hyaluronate prevents lidocaine-induced oxidative stress and mitochondrial dysfunction

To determine the pro-oxidant effects of lidocaine on the SW1353 cells, cells were treated with lidocaine and sodium hyaluronate, alone or in combination, and the intracellular ROS levels were then measured by flow cytometry using the ROS-sensitive fluorophore, DCF-DA. As indicated in the representative histogram, an increase in the ROS levels was indicated as a shift of cellular DCF fluorescence to the right. As shown in Figure 4A, treatment with final concentrations $(0.015 \%$ and $0.025 \%)$ of lidocaine for $72 \mathrm{~h}$ increased ROS production to approximately $21.9 \%$ and $27.1 \%$, respectively, whereas co-treatment with lidocaine and sodium hyaluronate attenuated it to approximately $2.5 \%$ and $6.5 \%$, respectively. These data indicate that sodium hyaluronate exerted a significant antioxidant effect, thus contributing cytoprotection against oxidative injury induced by lidocaine.

Next, we analyzed the integrity of mitochondrial function by flow cytometry using the fluorescent dye rhodamine- 123 . The results revealed that lidocaine induced a dose-dependent loss of mitochondrial membrane potential $(\Delta \Psi \mathrm{m})$ by decreasing the number of cells with intact $\Delta \Psi \mathrm{m}$ and increasing 


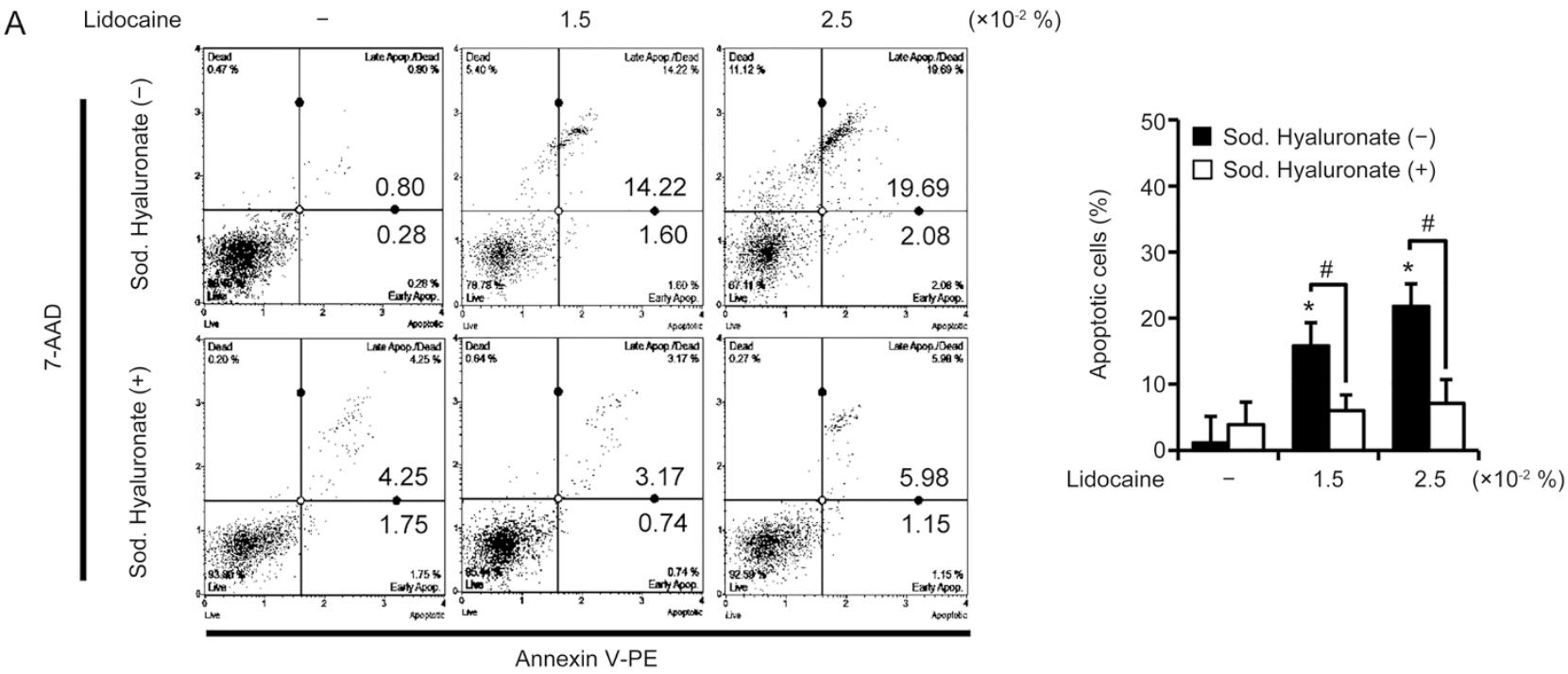

B

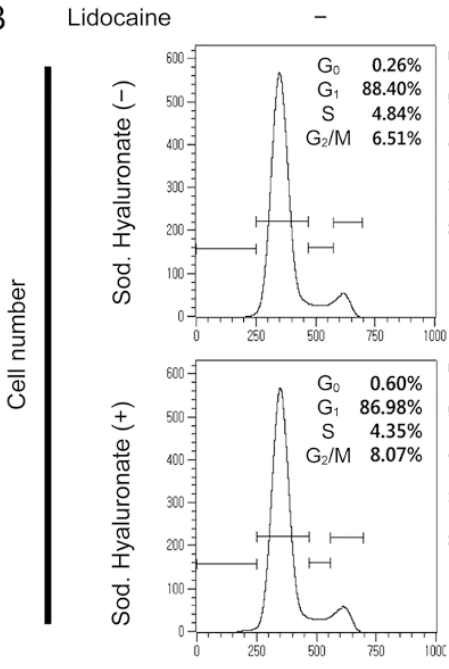

1.5

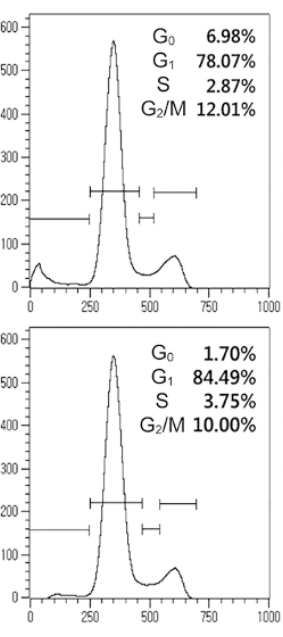

2.5

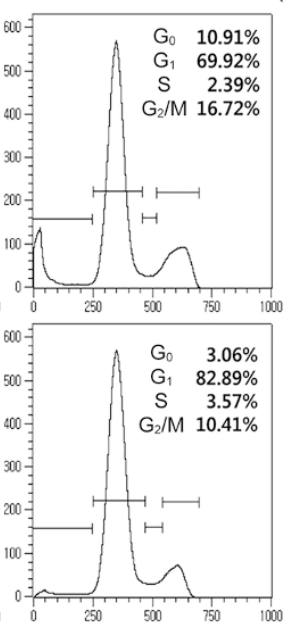

$\left(\times 10^{-2} \%\right)$

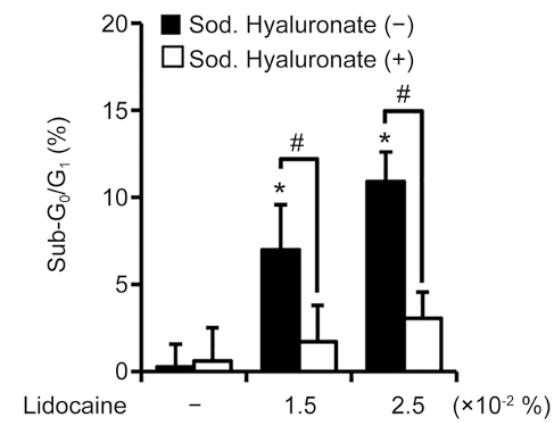

DNA content

Figure 3. The effects of sodium hyaluronate and lidocaine, alone or in combination, on apoptosis and the cell cycle in SW1353 cells. Cells were treated with sodium hyaluronate $(200 \mathrm{\mu g} / \mathrm{mL})$ and lidocaine $(0.015 \%$ and $0.025 \%)$, alone or in combination, for $72 \mathrm{~h}$. (A) The percentage of apoptotic cells after annexin $\mathrm{V}$ binding was obtained using a Muse cell analyzer. (B) Cells were analyzed using flow cytometry after having been stained with propidium iodide $(20 \mu \mathrm{g} / \mathrm{mL})$. The percentages of cell populations in sub- $G_{0} / G_{1}$ phase were calculated from three independent experiments. Mean $\pm S D$. $n=3$. ${ }^{*} P<0.05$ vs the control. ${ }^{\#} P<0.05$ between the two indicated groups.

the number of cells with low $\Delta \Psi \mathrm{m}$ after $72 \mathrm{~h}$ of exposure to varying concentrations of lidocaine. As shown in Figure $4 \mathrm{~B}$, compared with the control cells, lidocaine alone $(0.015 \%$ and $0.025 \%$ ) resulted in a significant decrease of fluorescence intensity by approximately $80.3 \%$ and $72.3 \%$, respectively. However, co-treatment with sodium hyaluronate restored the lidocaine-induced $\Delta \Psi \mathrm{m}$ decrease to approximately $97.4 \%$ and $94.6 \%$, respectively. These results revealed that the stability of $\Delta \Psi \mathrm{m}$ was significantly impaired by lidocaine, and sodium hyaluronate prevented mitochondrial disruption by blocking a substantial loss of $\Delta \Psi \mathrm{m}$ in SW1353 cells.
Sodium hyaluronate reduces lidocaine-induced chondrotoxicity through the suppression of $p 53$ activity

To find a possible effector molecule responsible for protecting the SW1353 cells against lidocaine-induced $\mathrm{G}_{2} / \mathrm{M}$ arrest and apoptosis, p53 expression was assayed by Western blotting. As shown in Figure 5A and 5B, increasing the concentration of sodium hyaluronate led to a dose-dependent decrease in both p53 protein and its transcription targets, including Bax and p21, whereas lidocaine treatment exhibited no significant changes on their levels. Co-treatment with lidocaine and sodium hyaluronate decreased the levels of p53, Bax, and 
A

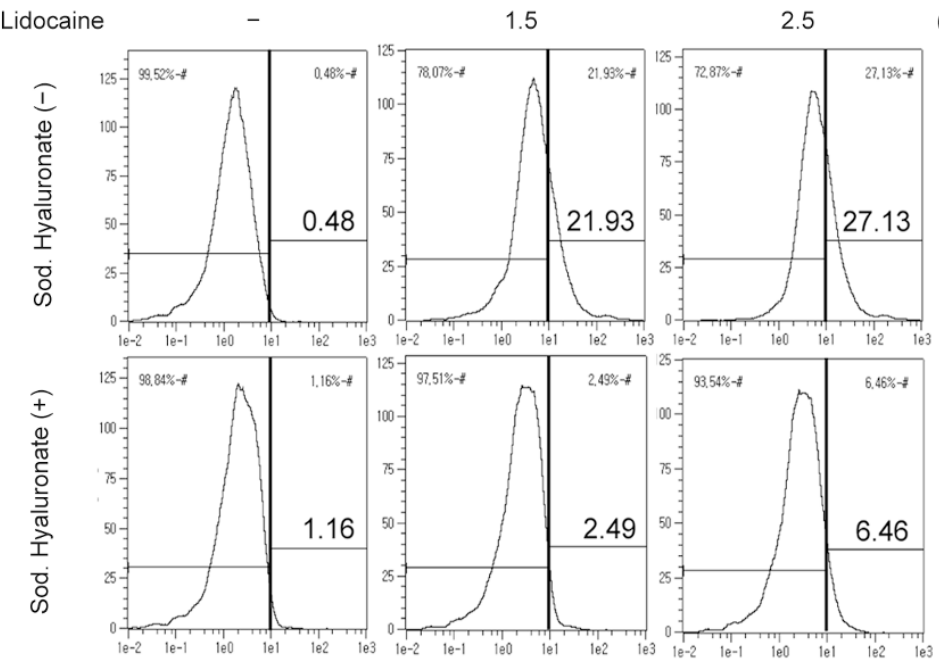

DCF-DA fluorescence $\left(\times 10^{-2} \%\right)$

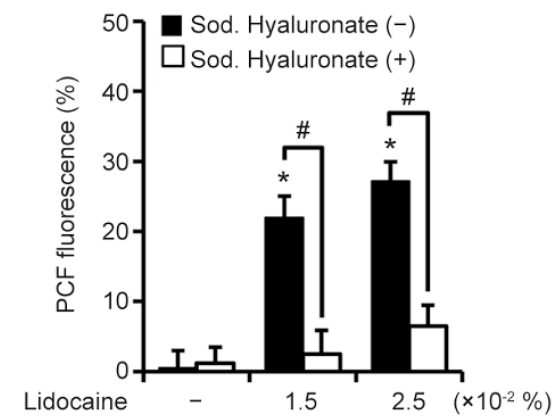

B

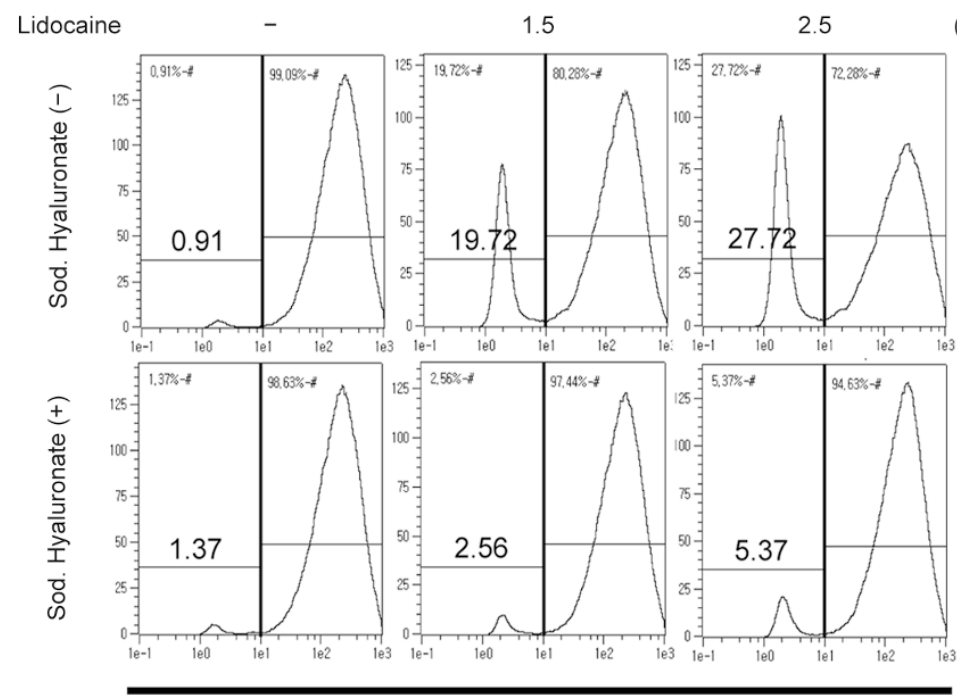

$\left(\times 10^{-2} \%\right)$

Fluorescence intensity

Figure 4. The effects of sodium hyaluronate and lidocaine, alone or in combination, on ROS level and mitochondrial membrane potential $(\Delta \Psi \mathrm{m})$. Cells were treated with sodium hyaluronate $(200 \mu \mathrm{g} / \mathrm{mL})$ and lidocaine $(0.015 \%$ and $0.025 \%)$, alone or in combination, for $72 \mathrm{~h}$. (A) The cellular ROS levels were measured using flow cytometry after having been stained with DCF-DA ( $10 \mu \mathrm{mol} / \mathrm{L})$. A shift of DCF fluorescence to the right indicates an increase of ROS. (B) The cells were stained with Rhodamine $123(30 \mathrm{nmol} / \mathrm{L})$. Mean \pm SD. $n=3$. ${ }^{*} P<0.05$ vs the control. ${ }^{\#} P<0.05$ between the two indicated groups.

p21 proteins (Figure 5C). Additionally, the proapoptotic/ antiapoptotic (Bax/Bcl-2) protein ratio, a marker for apoptosis, significantly increased in cells treated with lidocaine alone, whereas the addition of sodium hyaluronate effectively reversed it. To evaluate whether a decreased level of p53 is inhibits transcription, the cells were transiently transfected with a firefly luciferase reporter gene driven by the upstream p53 responsive element (p53RE-luc), prior to treatment. The luciferase activity data demonstrated that the transcriptional activation of p53RE-luc was suppressed by the combined treatment of the two compounds (Figure 5D). To ascertain a possible role of $\mathrm{p} 53$ in the protection provided by sodium hyaluronate against lidocaine-induced chondrotoxicity, the SW1353 cells were treated with p53-targeting siRNA and then examined for their sensitivity to the induction of apoptosis. The transfection of cells with p53-targeting siRNA led to a nearly complete inhibition of p53 protein expression (Figure 6A). Under the combined treatment of the two compounds, cytotoxicity was clearly prevented by p53 silencing but not by its nontargeting control siRNA (Figure 6B). These results suggest that sodium hyaluronate reduced lidocaine-induced chondrotoxicity, at least in part, through the suppression of p53 activity. 

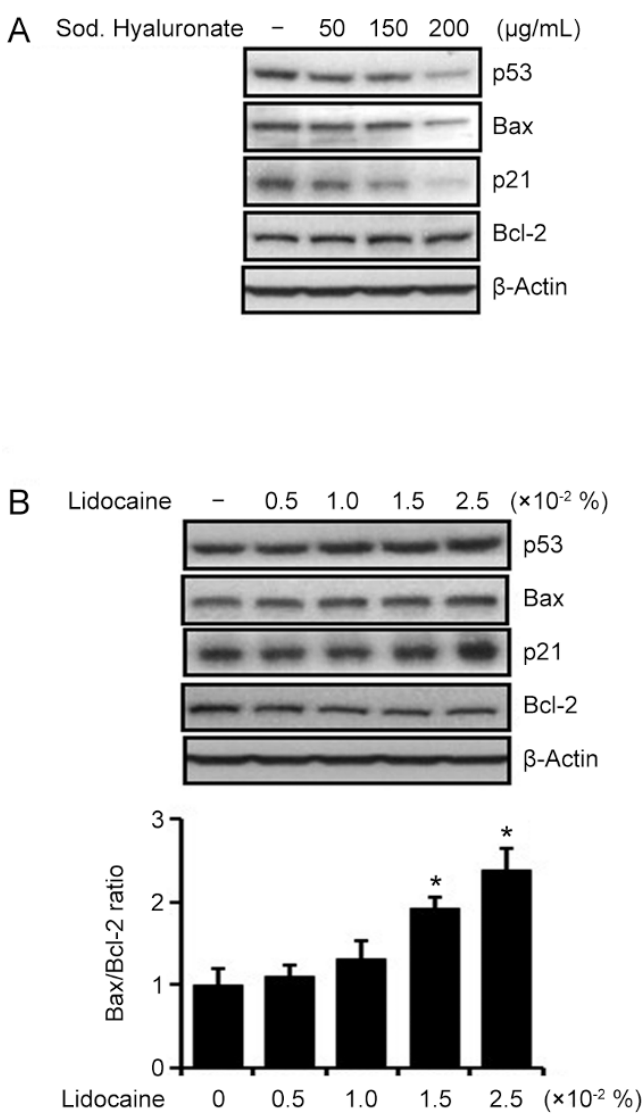
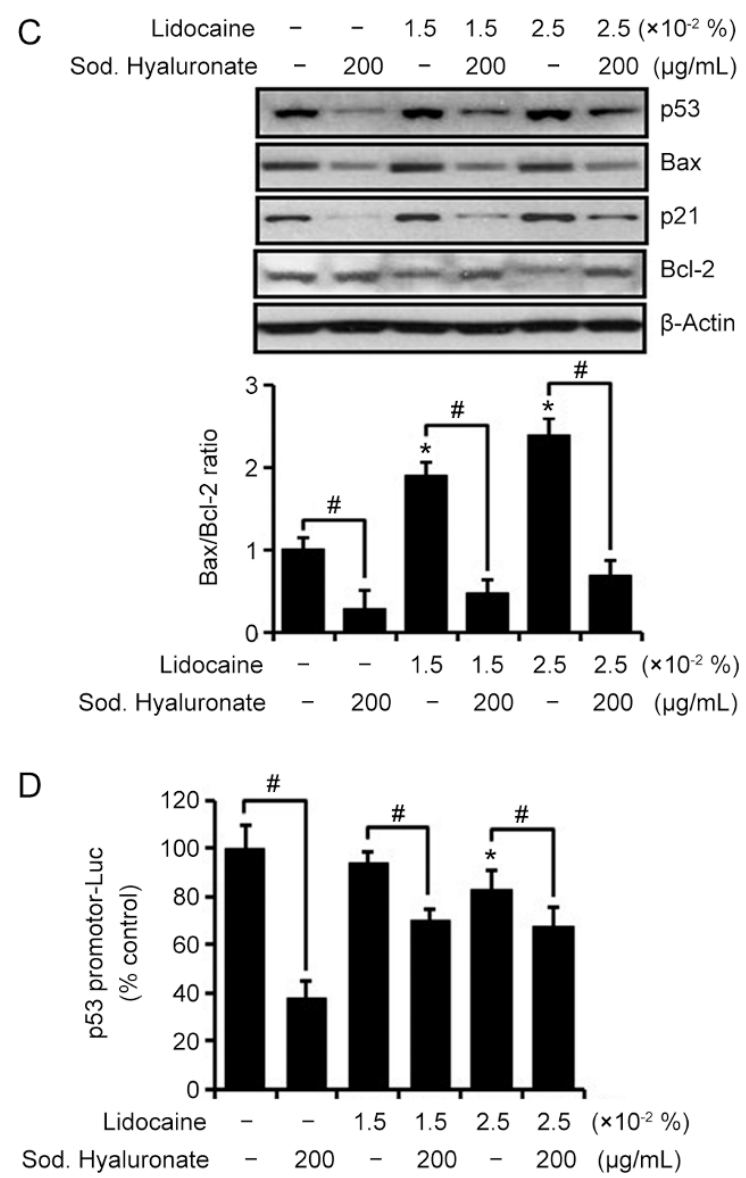

Figure 5. The effects of sodium hyaluronate and lidocaine, alone or in combination, on p53 and p53-regulated gene products in SW1353 cells. (A-C) Cells were treated with sodium hyaluronate (final, $0 \mu \mathrm{g} / \mathrm{mL}, 50 \mu \mathrm{g} / \mathrm{mL}, 150 \mu \mathrm{g} / \mathrm{mL}$, and $200 \mu \mathrm{g} / \mathrm{mL}$ ) (a), lidocaine (final, $0 \%, 0.005 \%, 0.01 \%, 0.015 \%$, and $0.025 \%$ ) (b), and sodium hyaluronate (final, $200 \mu \mathrm{g} / \mathrm{mL}$ ) and lidocaine (final, $0.015 \%$ and $0.025 \%$ ), alone or in combination (c), for $72 \mathrm{~h}$. Whole cell lysates were then analyzed by Western blotting with anti-p53, anti-Bax, anti-p21, and anti-Bcl-2. The blots were then stripped and re-probed with anti$\beta$-actin as a loading control. (D) Cells were transfected with a firefly luciferase reporter gene driven by the upstream p53 responsive element (p53REluc) for $24 \mathrm{~h}$ prior to incubation with sodium hyaluronate (final, $200 \mu \mathrm{g} / \mathrm{mL}$ ) and lidocaine (final, $0.015 \%$ and $0.025 \%$ ), alone or in combination, for another $72 \mathrm{~h}$, after which the luciferase activity in the cell lysates was determined. Mean \pm SD. $n=3 .{ }^{*} P<0.05$ vs the control. ${ }^{*} P<0.05$ between the two indicated groups.

Sodium hyaluronate protects mouse primary chondrocytes against lidocaine-induced toxicity and reduces p53 activity

To corroborate the above in vitro data, we harvested chondrocytes from the femoral condyles of newborn mice and cultured them ex vivo. Mouse articular chondrocytes were seeded in a 6-well culture plate at a density of $5 \times 10^{4}$ cells/ plate and reached $\sim 90 \%$ confluence by day 5 . The majority of cells exhibited a typical chondrocyte morphology with a rounded or polygonal shape (Figure 7A). However, lidocaine treatment increased the cytotoxicity in a dose-dependent manner, and elongated and fibroblast-like cells were observed (Figure 7B and 7C). Increasing the concentration of lidocaine induced apoptotic signaling, including a slight up-regulation of p53 and Bax, a down-regulation of Bcl-2, cleavage of caspase-3, and an increase in the Bax/Bcl-2 protein ratio (Figure $7 \mathrm{D}$ ). These responses were recovered by co-treatment with sodium hyaluronate.

\section{Discussion}

The goal of this paper was to study the protective roles of hyaluronan against lidocaine-induced chondrotoxicity. Investigating the underlying mechanisms of the antiapoptotic action of hyaluronan in SW1353 cells and mouse primary articular chondrocytes, we were able to identify three major findings. First, lidocaine increased chondrotoxicity because of the induction of apoptosis via ROS production and mitochondrial dysfunction. Second, sodium hyaluronate effectively reduced the amount of ROS induced by lidocaine. Third, sodium hyaluronate exerted an antiapoptotic action against lidocaine-induced chondrotoxicity, at least in part, by decreasing the level andactivity of the p53 protein, which executes central roles in ROS production, apoptosis, and cell cycle arrest. These results imply that the protective action of hyaluronan is due to antioxidant properties as well as an antiapoptotic effect.

Lidocaine, even at final concentrations $(0.015 \%$ and $0.025 \%)$ 
A

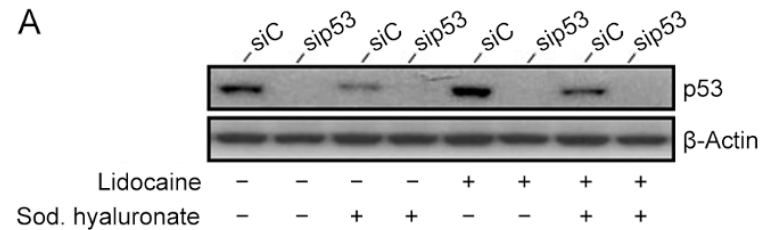

B

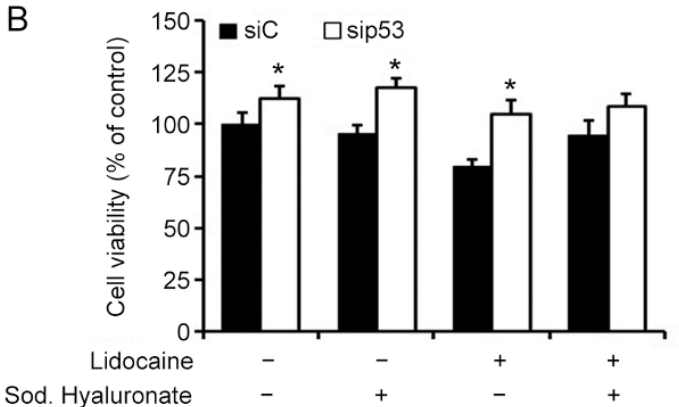

Figure 6. The effects of p53 knockdown on cell viability and p53 level in SW1353 cells. Cells were transfected with $10 \mathrm{nmol} / \mathrm{L}$ p53-targeting siRNA (sip53) or Stealth RNAi control ( $\mathrm{siC}$ ) for $24 \mathrm{~h}$ prior to incubation with sodium hyaluronate $(200 \mu \mathrm{g} / \mathrm{mL})$ and lidocaine $(0.015 \%$ and $0.025 \%$ ), alone or in combination, for an additional $72 \mathrm{~h}$, after which they were processed for Western blotting (A) or an MTT assay (B). The blots were then stripped and re-probed with anti- $\beta$-actin as a loading control. Mean \pm SD. $n=3$. ${ }^{*} P<0.05$ between the two indicated groups.

lower than the therapeutic concentration $(0.5 \%)$ for intra-articular injection, has growth-inhibiting and apoptosis-inducing effects on SW1353 cells. These effects were demonstrated by the increased chromatin condensation and nuclear fragmentation with enhanced cleavage of caspase- 3 , the appearance of a sub- $\mathrm{G}_{0} / \mathrm{G}_{1}$ peak in the flow cytometry assay, and the increased percentage of apoptotic cells in the annexin $\mathrm{V}$ binding assay. The induction of apoptosis by LAs in chondrocytes has been reported by several studies using in vitro cellular models ${ }^{[1,11]}$. Recently, Grishko et al have suggested that a mitochondrial pathway is involved in LA-induced apoptosis, which is associated with mitochondrial dysfunction resulting from damage to the mitochondrial genome ${ }^{[11]}$. Mitochondria, being the main site of ROS generation in the cell, are also a primary ROS target. Oxidative damage has been previously considered to be a possible mechanism for lidocaine-induced toxicity in the hippocampus and amygdala of adult rats ${ }^{[18]}$ as well as in rabbit corneal and lens tissues ${ }^{[19]}$. These findings are consistent with the results of this study, in which dose-dependent ROS level increases were demonstrated by a shift of cellular DCF fluorescence to the right in flow cytometric analysis. To the best of our knowledge, this ROS-induction effect in chondrocytes treated with lidocaine has not been reported previously. Increased ROS may contribute to mitochondrial damage and lead to cell death by acting as apoptotic signaling molecules ${ }^{[20]}$. A decrease in the mitochondrial membrane potential $(\Delta \Psi \mathrm{m})$ is recognized as an indicator of mitochondrial damage and is considered an early sign of apoptosis, leading to the efflux of cytochrome $c$ from the mitochondria, the sequential acti- vation of caspases, and eventually cell apoptosis ${ }^{[21]}$. In the results of this study, the effect of lidocaine on $\Delta \Psi \mathrm{m}$ and apoptosis in SW1353 cells seemed to be dependent on the excessive accumulation and production of ROS; thus, protection against these events may attenuate the lidocaine-induced damage. Sodium hyaluronate apparently exerted protection against lidocaine-induced toxicity, including ROS generation and $\Delta \Psi \mathrm{m}$ disruption. Exposure to sodium hyaluronate $(200 \mu \mathrm{g} / \mathrm{mL})$ for $72 \mathrm{~h}$ had little toxic effect on the SW1353 cells and significantly reduced the ROS induced by lidocaine. This implies that hyaluronan possesses antioxidant properties and exerts an antiapoptotic effect in SW1353 cells. The radical scavenging properties of hyaluronan have been demonstrated previously in various experimental models ${ }^{[22,23]}$.

An excess of ROS may lead to DNA damage, and these damaged cells subsequently undergo either cell cycle arrest to facilitate DNA repair or apoptosis to eliminate the excessively damaged cells ${ }^{[24]}$. The results of this study indicate that lidocaine induced an overall delay in cell cycle progression by affecting the $\mathrm{G}_{2} / \mathrm{M}$ phase of the cell cycle, and this effect could be a result of ROS production. The $\mathrm{G}_{2} / \mathrm{M}$ enrichment in the SW1353 cells exposed to lidocaine and its dependence on the lidocaine concentration, even below therapeutic doses, provide direct evidence of phase-specific, lidocaine-associated cell cycle regulation. Cell accumulation at the $\mathrm{G}_{2} / \mathrm{M}$ phase, along with apoptotic cell death, indicates that a common signal is sent to prevent damaged cells from further DNA replication and segregation, to drive irreversibly damaged cells into apoptosis, and to preserve genomic integrity. Several cellular effector molecules, including p53, are involved in arresting damaged cells at these checkpoints and inducing apoptosis. The p21 and Bax proteins are the accepted transcription targets of p53. The p53 protein leads to cell cycle arrest in either the $\mathrm{G}_{2} / \mathrm{M}$ or $\mathrm{G}_{0} / \mathrm{G}_{1}$ phase of the cell cycle by directly stimulating the expression of $\mathrm{p} 21^{\mathrm{WAF} 1 / \mathrm{CIP1}}$, an inhibitor of cyclin-dependent kinases $^{[25]}$. Elevated Bax protein levels accelerate the opening of mitochondrial pores for the release of cytochrome $c$ and induction of apoptotic cell death. In the present study, consistently with these observations, sodium hyaluronate downregulated the expression of p53, Bax, and p21 and prevented apoptosis and cell accumulation at the $\mathrm{G}_{2} / \mathrm{M}$ phase, which were related to the chondrotoxic activity of lidocaine. In particular, there was a marked decrease in the sub- $\mathrm{G}_{0} / \mathrm{G}_{1}$ fraction when p53 expression was decreased. This result indicated that p53 may participate in lidocaine-induced apoptosis as an event downstream from ROS production and mitochondrial damage. The p53 protein induces apoptosis because of its ability to bind specific DNA sequences and activate the transcription of target genes that encode proapoptotic proteins; this activity tilts the delicate balance between proapoptotic and antiapoptotic proteins in the favor of apoptosis. However, the effect of lidocaine on p53, Bax, and p21 was not obvious, as indicated by the analyses of their protein levels and by the p53 reporter gene assay. These findings imply that lidocaine may induce apoptosis in chondrocytes via a p53-independent pathway. Although the detailed molecular mechanism of the apoptosis- 
A
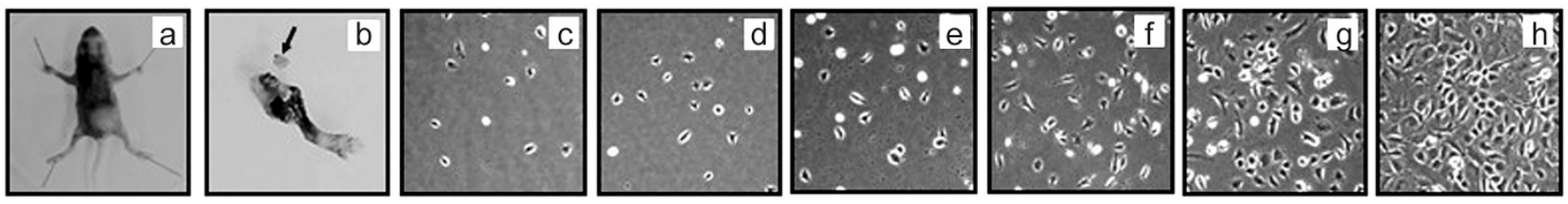

B

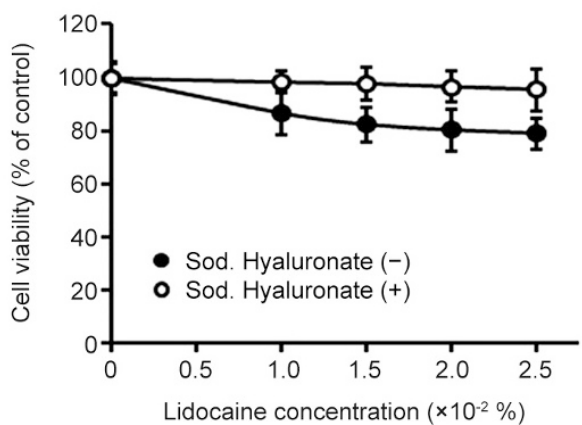

D

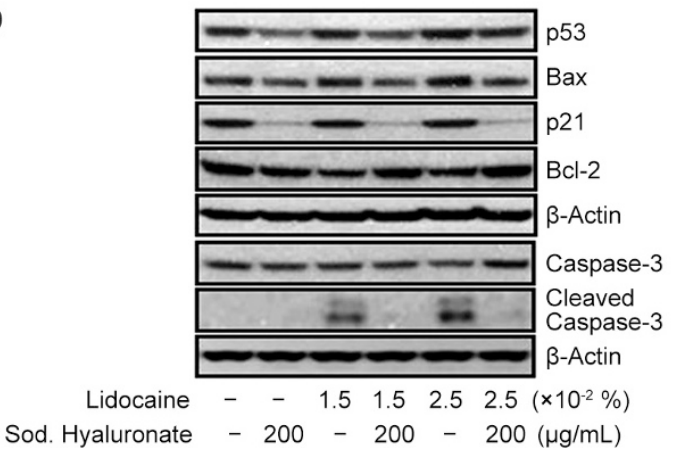

C
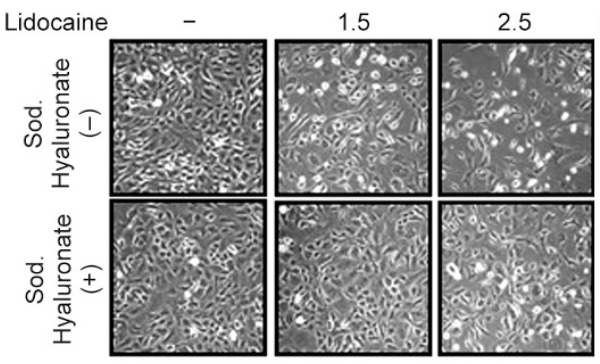

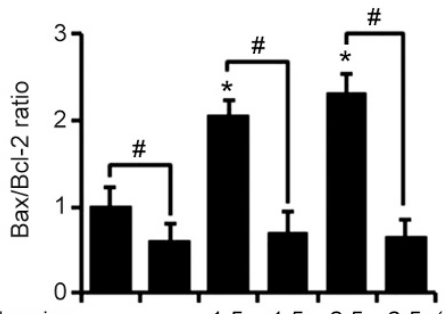

Lidocaine - $\quad \begin{array}{llll}1.5 & 1.5 & 2.5 & 2.5 \\ \left(\times 10^{-2} \%\right)\end{array}$

Sod. Hyaluronate $-200-200-200(\mu \mathrm{g} / \mathrm{mL})$

Figure 7. The effects of sodium hyaluronate and lidocaine, alone or in combination, on cell proliferation and apoptotic regulators in mouse primary articular chondrocytes. (A) Skin tissues were removed from the hind legs of 6-day-old mice, and the soft tissue of the joints was discarded to obtain the articular cartilage ( $\mathrm{a}$ and $\mathrm{b}$ ). The arrow indicates the articular cartilage. The morphology of mouse primary articular chondrocytes at $2 \mathrm{~h}(\mathrm{c}), 8 \mathrm{~h}(\mathrm{~d})$, $12 \mathrm{~h} \mathrm{(e),} 1$ day (f), 3 days (g), and 5 days of culture (h). (B) Cells were incubated with sodium hyaluronate $(200 \mu \mathrm{g} / \mathrm{mL})$ and the indicated concentrations of lidocaine, alone or in combination, for $72 \mathrm{~h}$. Cell viability was assessed by an MTT assay. Cells were incubated with sodium hyaluronate (200 $\mu \mathrm{g} / \mathrm{mL})$ and lidocaine $(0.015 \%$ and $0.025 \%)$, alone or in combination, for $72 \mathrm{~h}$. Cell morphology (C) and Western blot analysis (D). Mean \pm SD. $n=3$. ${ }^{*} P<0.05$ vs the control. ${ }^{\#} P<0.05$ between the two indicated groups.

inducing activity of lidocaine was not the focus of this study, other signaling pathway(s), including $\mathrm{Akt}^{[26]}, \mathrm{NF}-\mathrm{KB}^{[27]}$, and the activation of both extrinsic and intrinsic apoptosis pathways ${ }^{[28]}$ may also contribute to the lidocaine-induced chondrotoxicity. We cannot rule out whether lidocaine regulates these signaling pathways in the experimental conditions of this study; nevertheless, it can be postulated that lidocaine induces apoptosis by causing an increased ratio of Bax to Bcl-2. Alteration of the cellular Bax/Bcl-2 ratio, a marker of activation of the mitochondrial apoptotic pathway ${ }^{[29]}$, is more sensitive and reliable than the change in individual Bax or Bcl-2 levels. Bax is an apoptotic agonist, whereas Bcl-2 is an intracellular suppressor of apoptosis that prolongs cell survival. An increase in the Bax/Bcl-2 ratio may lead to mitochondrial dysfunction via mitochondrial membrane permeability, the release of cytochrome $c$, and activation of the caspase cascade. A significant dose-dependent increase in the Bax/Bcl-2 ratio after lidocaine treatment was also effectively reversed by co-treatment with sodium hyaluronate, thus shifting the balance toward Bcl-2 and leading to the inhibition of apoptosis induction. The results of the present study in SW1353 cells and mouse primary articular chondrocytes provide a theoretical basis for using hyaluronan to prevent lidocaine toxicity. However, further animal and clinical studies are required.

In summary, our study suggesting that lidocaine may cause cytotoxicity and cell cycle arrest by inducing oxidative mitochondrial damage. In addition, co-treatment with sodium hyaluronate allows chondrocytes to overcome the induction of cell death by maintaining low levels of p53, p21 and Bax and a high level of Bcl-2; co-treatment also protects against the induction of cell death in chondrocytes via the inhibition of ROS generation and mitochondrial depolarization. These results provide further insights for understanding the molecular basis of the antiapoptotic activity of hyaluronan in chondrocytes. Moreover, these results suggest that the use of hyaluronan as an adjuvant in the intra-articular injection of LAs may be beneficial in the prevention of chondrotoxicity. 


\section{Author contribution}

Sang-Han LEE and Soo A KIM designed the research and wrote the manuscript; Yoon-Jin LEE performed the experiments.

\section{References}

1 Rao AJ, Johnston TR, Harris AH, Smith RL, Costouros JG. Inhibition of chondrocyte and synovial cell death after exposure to commonly used anesthetics: chondrocyte apoptosis after anesthetics. Am J Sports Med 2014; 42: 50-8.

2 Zink W, Graf BM. Local anesthetic myotoxicity. Reg Anesth Pain Med 2004; 29: 333-40.

3 Chu CR, Izzo NJ, Papas NE, Fu FH. In vitro exposure to 0.5\% bupivacaine is cytotoxic to bovine articular chondrocytes. Arthroscopy 2006 ; 22: 693-9.

4 Karpie JC, Chu CR. Lidocaine exhibits dose- and time-dependent cytotoxic effects on bovine articular chondrocytes in vitro. Am J Sports Med 2007; 35: 1621-7.

5 Chu CR, Izzo NJ, Coyle CH, Papas NE, Logar A. The in vitro effects of bupivacaine on articular chondrocytes. J Bone Joint Surg $\mathrm{Br} 2008$; 90: 814-20.

6 Irwin W, Fontaine E, Agnolucci L, Penzo D, Betto R, Bortolotto S, et al. Bupivacaine myotoxicity is mediated by mitochondria. J Biol Chem 2002; 277: 12221-7.

7 Scholz A. Mechanisms of (local) anaesthetics on voltage-gated sodium and other ion channels. Br J Anaesth 2002; 89: 52-61.

8 Olschewski A, Olschewski H, Brau ME, Hempelmann G, Vogel W, Safronov BV. Effect of bupivacaine on ATP-dependent potassium channels in rat cardiomyocytes. Br J Anaesth 1999; 82: 435-8.

9 Nai GA, de Oliveira MC, de Oliveira Tavares G, Pereira LF, Soares ND, Silva PG. Evaluation of genotoxicity induced by repetitive administration of local anaesthetics: an experimental study in rats. Rev Bras Anestesiol 2015; 65: 21-6.

10 Bryant MS, Simmons HF, Harrell RE, Hinson JA. 2, 6-Dimethylanilinehemoglobin adducts from lidocaine in humans. Carcinogenesis 1994; 15: 2287-90.

11 Grishko V, Xu M, Wilson G, Pearsall AW. Apoptosis and mitochondrial dysfunction in human chondrocytes following exposure to lidocaine, bupivacaine, and ropivacaine. J Bone Joint Surg Am 2010; 92: 60918.

12 Ravikumar B, Sarkar S, Davies JE, Futter M, Garcia-Arencibia M, Green-Thompson ZW, et al. Regulation of mammalian autophagy in physiology and pathophysiology. Physiol Rev 2010; 90: 1383-435.

13 Jiang L, Sheikh MS, Huang Y. Decision making by p53: life versus death. Mol Cell Pharmacol 2010; 2: 69-77.

14 Mendoza G, Prieto JG, Real R, Perez M, Merino G, Alvarez Al. Antioxidant profile of hyaluronan: physico-chemical features and its role in pathologies. Mini Rev Med Chem 2009; 9: 1479-88.

15 Onur TS, Sitron CS, Dang A. Co-administration of hyaluronic acid with local anaesthetics shows lower cytotoxicity than local anaesthetic treatment alone in bovine articular chondrocytes. Bone Joint Res 2013; 2: 270-5.
16 Grishko V, Xu M, Ho R, Mates A, Watson S, Kim JT, et al. Effects of hyaluronic acid on mitochondrial function and mitochondria-driven apoptosis following oxidative stress in human chondrocytes. J Biol Chem 2009; 284: 9132-9.

17 Gosset M, Berenbaum F, Thirion S, Jacques C. Primary culture and phenotyping of murine chondrocytes. Nat Protoc 2008; 3: 1253-60.

18 Cano-Europa E, López-Galindo GE, Hernández-García A, Blas-Valdivia V, Gallardo-Casas CA, Vargas-Lascari M, et al. Lidocaine affects the redox environment and the antioxidant enzymatic system causing oxidative stress in the hippocampus and amygdala of adult rats. Life Sci 2008; 83: 681-5.

19 Demir MN, Demir ZA, YalçinTök O, Yilmaz FM, Yilmaz G, Nurözler AB, et al. Oxidative stress of intracameral lidocaine and levobupivacaine on ocular tissues. Br J Ophthalmol 2010; 94: 1083-7.

20 Vento MT, Zazzu VZ, Loffreda A, Cross JR, Downward J, Stoppelli MP, et al. Praf2 is a novel $\mathrm{Bcl}-\mathrm{xL} / \mathrm{Bcl}-2$ interacting protein with the ability to modulate survival of cancer cells. PLoS One 2010; 5: e15636.

21 Wang W, Zhang Z, Guo Y, Dong Y, Huang X, Zhou Y, et al. Carbon disulfide induces mitochondria-mediated apoptosis in Sertoli-germ cells coculture. Mol Cell Toxicol 2015; 11: 175-85.

22 Suzuki Y, Yamaguchi T. Effects of hyaluronic acid on macrophage phagocytosis and active oxygen release. Agents and Actions 1993; 38: 32-7.

23 Moseley R, Walker M, Waddington RJ. Comparison of the antioxidant properties of wound dressing materials-carboxymethylcellulose, hyaluronan benzyl ester and hyaluronan, towards polymorphonuclear leukocyte-derived reactive oxygen species. Biomaterials 2003; 24: 1549-57.

24 Kim SH, Ko JW, Koh SK, Lee IC, Son JM, Moon CJ, et al. Silver nanoparticles induce apoptotic cell death in cultured cerebral cortical neurons. Mol Cell Toxicol 2014; 10: 173-9.

25 Bishayee K, Khuda-Bukhsh AR, Huh SO. PLGA-Loaded GoldNanoparticles Precipitated with Quercetin Downregulate HDAC-Akt Activities Controlling Proliferation and Activate p53-R0S Crosstalk to Induce Apoptosis in Hepatocarcinoma Cells. Mol Cells 2015; 38: 518-27.

26 Maurice JM, Gan Y, Ma FX, Chang YC, Hibner M, Huang Y. Bupivacaine causes cytotoxicity in mouse $\mathrm{C} 2 \mathrm{C} 12$ myoblast cells: involvement of ERK and Akt signaling pathways. Acta Pharmacol Sin 2010; 31: 493500.

27 Liu J, Zhang H, Qi Z, Zheng X. Lidocaine protects against renal and hepatic dysfunction in septic rats via downregulation of Toll-like receptor 4. Mol Med Rep 2014; 9 :118-24.

28 Iwasaki K, Sudo H, Yamada K, Ito M, Iwasaki N. Cytotoxic effects of the radiocontrast agent iotrolan and anesthetic agents bupivacaine and lidocaine in three-dimensional cultures of human intervertebral disc nucleus pulposus cells: identification of the apoptotic pathways. PLoS One 2014; 9: e92442.

29 Kim YS, Li XF, Kang KH, Ryu B, Kim SK. Stigmasterol isolated from marine microalgae Naviculaincertainduces apoptosis in human hepatoma HepG2 cells. BMB Rep 2014; 47: 433-8. 